\title{
IL-2 Mutein/Fc Fusion Protein AMG 592
}

National Cancer Institute

\section{Source}

National Cancer Institute. IL-2 Mutein/Fc Fusion Protein AMG 592. NCI Thesaurus. Code C147121.

A recombinant fusion protein consisting of a variant form of the human cytokine interleukin 2 (IL-2; IL2), IL-2 mutein, fused to a human Fc molecule, with potential cellprotective and anti-inflammatory activities. Upon intravenous administration of recombinant human IL-2 mutein/Fc fusion protein AMG 592, the IL-2 mutein moiety binds to its receptors, and activates IL-2-mediated signaling pathways. IL-2 mutein preferentially expands and activates regulatory $\mathrm{T}$-cells (Tregs), thereby preventing inadequate immune responses and restoring immune homeostasis. This may help control or prevent inflammatory reactions as seen in inflammatory-based diseases, such as autoimmune diseases and graft-versus-host disease (GVHD). IL-2 mutein is less able to stimulate natural killer (NK) cells and conventional T-cells, which may lead to reduced toxicity compared to treatment with recombinant IL-2. Fusion to the Fc increases the stability and half-life of AMG 592. Tregs play a key role in the prevention of inflammatory diseases and immune homeostasis. 